\title{
SYNTHESIS OF ZN - CATALYZED SUBSTITUTED -3-(3, 4, 5- TRIMETHOXYPHENYL) PROP-2-EN-1-KETO-TRIAZOLES AND THEIR BIOLOGICAL EVALUATION
}

\author{
Dhondiba Vishwanath ${ }^{1}$, Devaraju Kesagodu, ${ }^{2 *}$, P. Ranjini $^{3}$, S. Vijayakumar ${ }^{4}$ \\ and Y.B. Basavaraju ${ }^{5}$ \\ ${ }^{1-2}$ Department of Chemistry Yuvaraja'sCollege,University of Mysore, \\ Mysuru-570005, Karnataka, India. \\ ${ }^{3}$ Department of Biotechnology,Maharani's ScienceCollege,University of Mysore, \\ Mysuru-570005, Karnataka, India. \\ ${ }^{4}$ Department of Chemistry,GCW, Kolar, University of Bangalore, \\ Bengaluru-563101, Karnataka, India. \\ ${ }^{5}$ Department of Chemistry,University of Mysore, Mysuru-570005, Karnataka, India. \\ *E-mail : yuvadevaraj2018@gmail.com
}

\begin{abstract}
The importance of azide and chalcones is highly potential and potent in biology and chemistry. Therefore we Synthesized triazole derivatives of substituted -3-(3, 4, 5-trimethoxyphenyl) prop-2-en-1-one in presence of Zn catalyst in alcohol in good yields by chalcone method. All synthesized compounds were characterized by spectral techniques. Further, the compounds were investigated for biological activities

Keywords: chalcone, triazole, sodium azide, zinc metal, Claisen -Schmidt condensation, biological activity

(C) RASĀYAN. All rights reserved
\end{abstract}

\section{INTRODUCTION}

There is well known pharmaceutical application of synthetic drugs is based on the nucleus of the compound such that important nucleus containing compounds are chalcones. Chalcones are widely occurring compounds in the plant kingdom. They exhibit various types of biological activity. The chalcones (trans-1, 3-diaryl-2-propen-1-ones) (Fig.-1) is $\alpha, \beta$-unsaturated ketones 1,2,3,4 containing two aromatic rings with different substituent's, to increase the activity. Hence modifications in chalcone structure are required to reduce its toxicity and to enhance it's biological activity ${ }^{4}$. The medicinally active and less cytotoxic new nitrogen heterocyclic intermediates of chalcone have been synthesized. The modification of the chalcone structure might enhance the biological activity with favorable solubility and reduced toxicity ${ }^{5,6}$. Some synthesized analogs of chalcone showed better antibacterial anti viralanti fungal and anti neoplastic activity. The structures of the synthesized new chalconetriazole compounds were confirmed by IR, 1H-NMR, 13C-NMR and Mass spectral data. They will be screened for biological activities.

\section{Chemistry}

The literature survey shows that chalcone containing hetero group like Nitrogen exhibits very good antibiological activities. ${ }^{1,3,5,7,8-11}$ The presence of unsaturation is found to be responsible for their biological activity. Triazole is a five-membered heterocyclic which contains three nitrogen heteroatoms. The triazole nucleus, ${ }^{12-15}$ is one of the most important and well-known heterocycles which is a common and integral feature of a variety of natural products and medicinal agents. Triazole nucleus is present as a core structural component in an array of drug categories such as antimicrobial, anti-inflammatory, analgesic, antiepileptic, antiviral, antineoplastic, antihypertensive, anti-malarial, local anesthetic, anti-anxiety,

Rasayan J. Chem., 11(3), 955-961(2018)

http://dx.doi.org/10.31788/RJC.2018.1133014

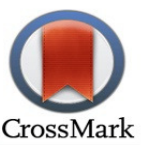


antidepressant, antihistaminic, antioxidant, anti-tubercular, anti-Parkinson's, anti-diabetic, anti-obesity and immunomodulatory agents, etc. ${ }^{16,17}$

The broad and potent activity of triazole and their derivatives has established them as pharmacologically significant scaffolds. The basic heterocyclic rings present in the various medicinal agents are 1, 2, 3triazole and 1,2, 4-triazole. A large volume of research has been carried out on triazole and their derivatives, which has proved the pharmacological importance of this heterocyclic nucleus. These compounds provide structural precedence that our chalcone and triazole analogs may lead to the generation of novel biologically active pharmaceutical drugs. The present paper is an attempt to review the pharmacological activities reported for triazole derivatives in the current literature with an update of recent research findings on this nuclei. So we planned and synthesized the triazole derivatives using which very effective against the pathogens.

\section{EXPERIMENTAL}

\section{Material and Methods}

All the reagents and chemicals were purchased from Merck chemicals used without further purification. Melting points were taken in open capillary tubes and are uncorrected. TLC is performed with E. Merck precoated silica gel plates (60F-254) with iodine as a spot developing agent. Acme, India silica gel, 60120 mesh is used for column chromatography. IR spectra in $\mathrm{KBr}$ were recorded on Perkin-Elmer model 683 spectrometers. 1H NMR (400 MHz) and 13C NMR (100 MHz) spectra were recorded CDCl3 solvent containing tetra methyl silane (TMS) as internal references were recorded on Bruker spectrometer; Elemental analyses were performed on a PerkinElmer 2400. Mass spectra were obtained by Water-QTOF ultima spectrometer. Micro analytical data were obtained by elemental-Vario EL-III (Scheme-1).

\section{Synthesis of Substituted -3-(3, 4, 5-trimethoxyphenyl) prop-2-en-1-one 4(a-f) General Procedure for the Synthesis of Chalcones}

A mixture of acetophenone 14 (a-d) 0.01 mole and 3, 4, 5-trimethoxybenzaldehyde 0.01 mole were stirred at room temperature in an ethanolic solution of potassium hydroxide for 2-3 hours. The formed yellowish crystals were filtered off washed with distilled water, dried and recrystallized from ethanol to give the product as yellow crystals.

(E)-1-(3, 4-dimethoxyphenyl)-3-(3, 4, 5-trimethoxyphenyl) prop-2-en-1-one (3a)

Color: light yellow solid. Yield: 80.10\%. Mp: 98- $100{ }^{\circ} \mathrm{C}$. IR: 3164-2956 (Ar.-CH), 1671 (C=O), 1545 $(\mathrm{C}=\mathrm{C})$; 1H NMR: 8.01 (1 H, d, J = 8.2, $\beta$-CH), 7.67-7.12 (4 H, m, Ar.-H), 6.70(2 H, s, Ar.-CH), 7.51 (1 $\mathrm{H}, \mathrm{d}, \mathrm{J}=8.2, \alpha-\mathrm{CH}), 3.82$ (15 H, s, OCH3); 13C NMR: 196.7, 155.5, 153.2, 150.3, 145.3, 130.3, 126.4, 123.2, 122.3, 112.7, 107.5, 60.8, 56.1; MS, m/z: 358.14 (M+). C20H22O6: C, 67.03; H, 6.19 O, 26.79. Found: C, 67.05; H, 6.18; O, 26.78\%.

(E)-1-(3, 4-dimethylphenyl)-3-(3, 4, 5-trimethoxyphenyl) prop-2en-1-one (3b)

Color: colorless solid. Yield: 77.50\%. Mp: 104-105 ${ }^{\circ} \mathrm{C}$. IR: 3065-2925 (Ar.-CH), 1663 (C=O), 1539 $(\mathrm{C}=\mathrm{C})$; 1H NMR: 7.87-6.91 (5 H, m, Ar.-H), 8.06 (1 H, d, J = 8.3, $\beta-\mathrm{CH}), 6.78(2 \mathrm{H}, \mathrm{s}, \mathrm{Ar} .-\mathrm{CH}), 7.62(1$ $\mathrm{H}, \mathrm{d}, \mathrm{J}=8.2, \alpha-\mathrm{CH}), 3.83$ (9 H, s, OCH3); 13C NMR: 189.7, 153.0, 145.1, 138.4, 137.3, 136.3, 132.6, 130.0, 126.4, 121.3, 103.8, 60.8, 56.1, 18.8; MS, m/z: 326.15(M+). C20H22O4: C, 73.60; H, 6.79; O, 19.61. Found: C, 73.65; H, 6.76; O, 19.59\%.

(E)-1-(3-chloro, 4-methylphenyl)-3-(3, 4, 5-trimethoxyphenyl) prop-2-en1-one (3c) Color: yellow solid. Yield: 81.30\%. Mp: 109-110 ${ }^{\circ} \mathrm{C}$. IR: 3159-2957 (Ar.-CH), 1675 (C=O), 1549 $(\mathrm{C}=\mathrm{C})$; 1027(Ar.-Cl); 1H NMR: 8.10-6.92 (5 H, m, Ar.-H), $8.05(1 \mathrm{H}, \mathrm{d}, \mathrm{J}=8.1, \beta-\mathrm{CH}), 7.58(1 \mathrm{H}, \mathrm{d}, \mathrm{J}=$ 8.1, $\alpha-\mathrm{CH}), 3.88$ (12 H, s, OCH3); 13C NMR: 189.7, 160.4, 153.0, 145.1, 138.4, 131.6, 130.9, 129.0, 126.4, 121.3, 112.6, 103.8, 60.8, 56.1, 55.6; MS, m/z: $362.80(\mathrm{M}+)$. C19H19ClO5: C, 62.90; H, 5.28; Cl, 9.77; O, 22.05. Found: C, 62.86; H, 5.25; Cl, 9.79; O, 22.10\%.

(E)-1-(3, 4-dihydroxy)-3-(3, 4, 5-trimethoxyphenyl) prop-2-en-1-one (3d)

Color: colorless solid. Yield: 85.90\%. Mp: 95-97 ${ }^{\circ} \mathrm{C}$. IR:3480-3500 ( Ar.- OH), 3150- 2972 (Ar.-CH), $1677(\mathrm{C}=\mathrm{O}), 1543(\mathrm{C}=\mathrm{C})$; 1H NMR: $9.48(2 \mathrm{H}, \mathrm{s}, \mathrm{Ar} .-\mathrm{OH}) ; 8.05(\mathrm{~d}, 1 \mathrm{H}, \mathrm{J}=7.8, \beta-\mathrm{CH}), 7.89-7.68(9 \mathrm{H}$, m, Ar.-H), 7.59 (1 H, d, J = 7.8, $\alpha-C H) ; 3.83$ (9H, s, OCH3); 13C NMR: 196.1, 153.1, 153.0 145.1, 138.4, 
126.4, 123.9, 123.2, 121.3, 117.8, 117.0, 103.8, 60.8,56.1; MS, m/z: 330.11 (M+). C18H18O6: C, 65.45; H, 5.49; O, 29.06. Found: C, 65.34; H, 5.53; O, 29.13\%

SCHEME

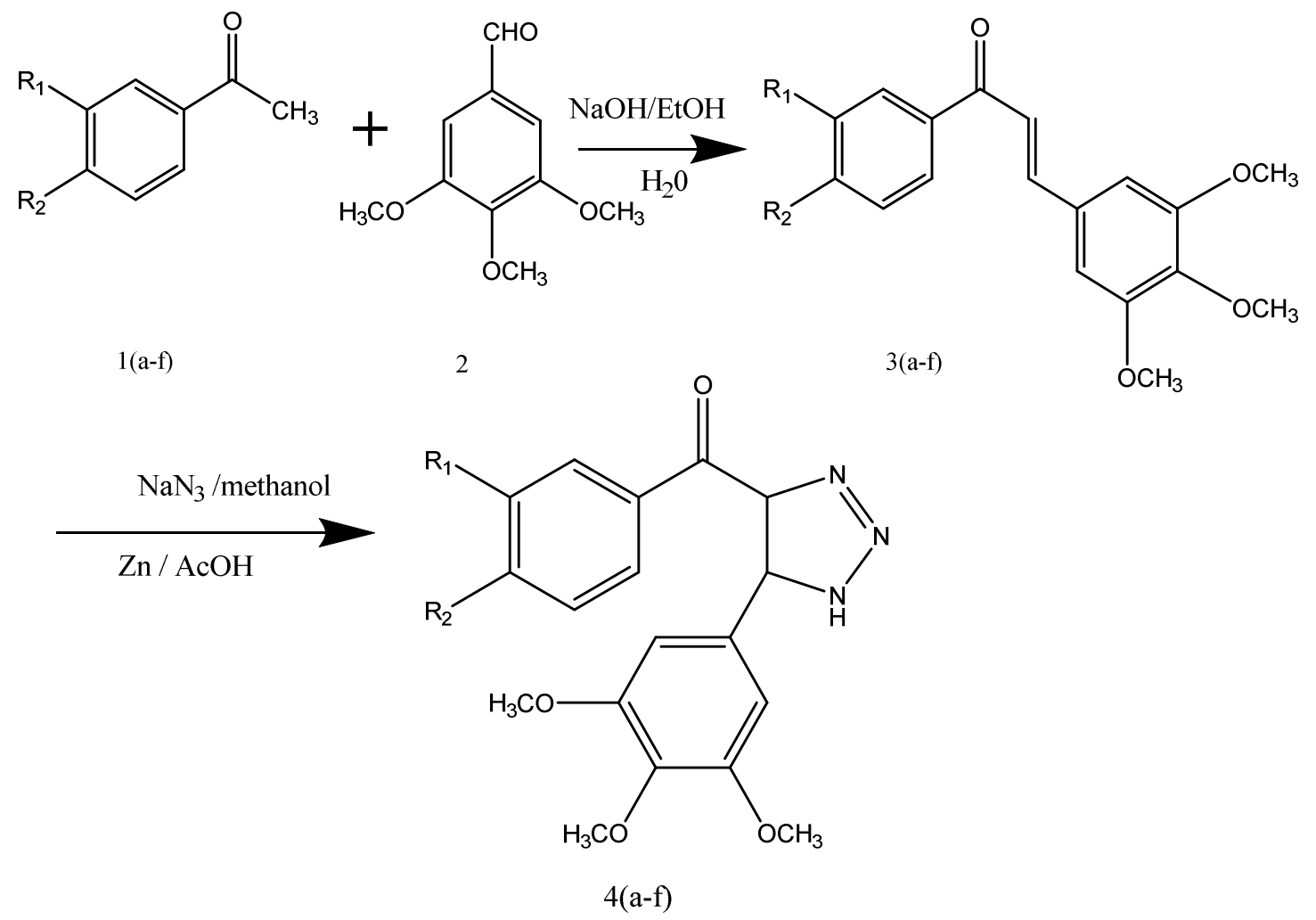
(a) $\mathrm{R}_{1}=\mathrm{OCH}_{3} \quad \mathrm{R}_{2}=\mathrm{OCH}_{3}$
(b) $\mathrm{R}_{1}=\mathrm{CH}_{3} \quad \mathrm{R}_{2}=\mathrm{CH}_{3}$
(c) $\mathrm{R}_{1}=\mathrm{Cl} \quad \mathrm{R}_{2}=\mathrm{OCH}_{3}$
(d) $\mathrm{R}_{1}=\mathrm{OH} \quad \mathrm{R}_{2}=\mathrm{OH}$
(e) $\mathrm{R}_{1}=\mathrm{NH}_{2} \quad \mathrm{R}_{2}=\mathrm{H}$
(f) $\mathrm{R}_{1}=\mathrm{NO}_{2} \quad \mathrm{R}_{2}=\mathrm{H}$

Fig-1: Protocol for the Synthesis of ChalconeTriazoles 4(a-f)

(E)-1-(3-amino)-3-(3, 4, 5-trimethoxyphenyl) prop-2-en-1-one (3e)

Color: light brown solid. Yield: 84.25\%. Mp: 101-103 ${ }^{\circ} \mathrm{C}$. IR: 3420-3509( NH), 3150- 2972 (Ar.-CH), $1677(\mathrm{C}=\mathrm{O}), 1543(\mathrm{C}=\mathrm{C})$; 1H NMR: $8.02(\mathrm{~d}, 2 \mathrm{H}, \mathrm{J}=7.1, \beta-\mathrm{CH}), 7.30-6.91(6 \mathrm{H}, \mathrm{m}, \mathrm{Ar} . \mathrm{H}), 8.06(1 \mathrm{H}, \mathrm{d}$, $\mathrm{J}=7.8, \alpha-\mathrm{CH}) ; 5.28(2 \mathrm{H}, \mathrm{s}, \mathrm{Ar}-\mathrm{NH} 2), 3.85$ (9H, s, OCH3); 13C NMR: 189.7, 153.0, 148.9, 145.1, 138.7, 138.4, 134.3, 126.4, 121.3, 120.8, 118.5, 114.2, 103.8, 60.8,56.1; MS, m/z: 313.13 (M+). C18H19NO4: C, 69.00; H, 6.11; N, 4.49; O, 20.40. Found: 68.95; H, 6.08; N, 4.55; O, $20.42 \%$.

\section{(E)-1-(3- nitro)-3-(3, 4, 5-trimethoxyphenyl) prop-2-en-1-one (3f)}

Color: yellow solid. Yield: 81.35\%. Mp: 107-108 ${ }^{\circ} \mathrm{C}$. IR: 3150- 2972 (Ar.-CH), 1677 (C=O), 1543 $(\mathrm{C}=\mathrm{C}) ; 1455(\mathrm{Ar}-\mathrm{NO} 2)$; 1H NMR: $8.06(\mathrm{~d}, 1 \mathrm{H}, \mathrm{J}=7.6, \beta-\mathrm{CH}), 8.54-6.91(9 \mathrm{H}, \mathrm{m}, \mathrm{Ar} . \mathrm{H}), 7.60(1 \mathrm{H}, \mathrm{d}, \mathrm{J}=$ 7.8, $\alpha-\mathrm{CH}), 3.83$ (9H, s, OCH3); 13C NMR: 189.7, 153.0, 148.4, 145.1, 138.8, 138.4, 134.6, 130.1, 129.7, 
RASĀYAN J. Chem.

Vol. 11 | No. 3 |955 - 961 | July - September | 2018

126.4, 123.5, 121.3, 103.8, 60.8,56.1; MS, m/z: 343.11 (M+).C18H17NO6: C, 62.97; H, 4.99; N, 4.08; O, 27.96. Found: C, 62.95; H, 4.95; N, 4.10; O, 28.00\%

General procedure for the preparation of triazole derivatives of $(3,4$ substituted $)-[5-(3,4,5-$ trimethoxyphenyl)-4, 5-dihydro-1H-1, 2, 3-triazoles

Freshly prepared zinc-copper couple 0.5 mole was taken in a round-bottomed flask which was fitted with a condenser, dropping funnel and magnetic stirrer. $50 \mathrm{ml}$ of ether was added to the dry $\mathrm{Zn}-\mathrm{Cu}$ couple followed by a few milliliters of the dichloromethane. The reaction was started immediately which was indicated by bubbles rising from the couple while the stirred suspension was kept at gentle reflux for two minutes. A mixture of chalcone 0.25 mole and the remaining dichloromethane 0.35 mole was added dropwise for 2 hours. The reaction mixture was refluxed for 20-30 hours. After completion of the reaction, the ether solution was decanted slowly from the unchanged

the mixture was poured in water and extracted with ethyl acetate, washed with $\mathrm{Na}_{2} \mathrm{CO}_{3}$ solution, dried with anhydrous $\mathrm{Na}_{2} \mathrm{SO}_{4}$, Then, the solvent evaporated to dryness, to give crude product $4(\mathrm{a}-\mathrm{f})$. The products were recrystallized using ethanol.

E (3, 4-dimethoxyphenyl) - [5-(3, 4, 5-trimethoxyphenyl)-4, 5-dihydro-1H-1, 2, 3-triazol 4-yl] methanone(4a)

Colour: Yellow solid. M.P. $123-125{ }^{\circ} \mathrm{C}$; Yield: $75.7 \%$, IR: IR $(\mathrm{KBr}): 1727 \mathrm{~cm}^{-1}(\mathrm{C}=\mathrm{O}), 1620 \mathrm{~cm}^{-1}$ $(\mathrm{N}=\mathrm{N}),{ }^{1} \mathrm{H}-\mathrm{NMR}\left(\mathrm{CDCl}_{3}\right) ; \delta(\mathrm{ppm}): 2.3(\mathrm{~s}, 1 \mathrm{H}, \mathrm{NH}) ; 3.3\left(\mathrm{~d}, 2 \mathrm{H}, \mathrm{CH}_{2}, \operatorname{adj} . \mathrm{N}=\mathrm{N}\right) ; 3.71-3.85(\mathrm{bs}, 15 \mathrm{H}$, $\left.\mathrm{OCH}_{3}\right), 4.4\left(\mathrm{~d}, 2 \mathrm{H}, \mathrm{CH}_{2}\right.$, adj $\left.\mathrm{NH}\right)$ and $6.65-7.62(\mathrm{~m}, 5 \mathrm{H}, \mathrm{Ar} . \mathrm{H}) .{ }^{13} \mathrm{C}-\mathrm{NMR}\left(\mathrm{CDCl}_{3}\right) ; \delta(\mathrm{ppm}): 56.1,60.8$, 69.9, 81.9, 102.1, 110.3, 111.7, 122.1, 130.0, 137.2, 137.8, 149.7, 152.7, 154.2, 197.6. Mass (m/z): 401.6, Elemental Analysis (\%): For $\mathrm{C}_{20} \mathrm{H}_{23} \mathrm{~N}_{3} \mathrm{O}_{6}$, Calculated: C, 59.84; H, 5.78; N, 10.47; O, 23.91. Found: C, 59.78; H, 5.76; N, 10.48; O, 23.98.

E (3, 4 - dimethylphenyl) [5-(3, 4, 5-trimethoxyphenyl)-4, 5-dihydro-1H-1, 2, 3-triazol-4-yl] methanone(4b)

Colour: light yellow solid. M.P. $119-120{ }^{\circ} \mathrm{C}$; Yield: 73.7\%, IR: IR (KBr): $1661 \mathrm{~cm}^{-1}(\mathrm{C}=\mathrm{O}), 1630-1575$ $\mathrm{cm}^{-1}\left(\mathrm{~N}=\mathrm{N},{ }^{1} \mathrm{H}-\mathrm{NMR}\left(\mathrm{CDCl}_{3}\right) ; \delta(\mathrm{ppm}): 2.31-2.32\left(\mathrm{~d}, 6 \mathrm{H}, \mathrm{CH}_{3}, \mathrm{NH}\right) ; 3.3\left(\mathrm{~d}, 2 \mathrm{H}, \mathrm{CH}_{2}\right.\right.$, adj. $\left.\mathrm{N}=\mathrm{N}\right) ; 3.71-$ 3.85 (bs, 9H, $\left.\mathrm{OCH}_{3}\right), 4.4\left(\mathrm{~d}, 2 \mathrm{H}, \mathrm{CH}_{2}\right.$, adj. $\left.\mathrm{NH}\right)$ and $6.65-7.73(\mathrm{~m}, 5 \mathrm{H}, \mathrm{Ar} .-\mathrm{H}) .{ }^{13} \mathrm{C}-\mathrm{NMR}\left(\mathrm{CDCl}_{3}\right) ; \delta$ (ppm): 18.8, 56.1, 60.8, 69.9, 81.1, 102.1, 122.9, 129.0, 133.0, 137.2, 139.3, 142.2, 152.7, 197.6. Mass $(\mathrm{m} / \mathrm{z})$ : 369.17, Elemental Analysis (\%): For $\mathrm{C}_{20} \mathrm{H}_{23} \mathrm{~N}_{3} \mathrm{O}_{4}$, Calculated: C, 65.03; H, 6.28; N, 11.37; O, 17.32. Found: C, 65.13; H, 6.33; N, 11.29; O, 17.12.

E (3-Chloro- 4 -methoxyphenyl) [5-(3, 4, 5-trimethoxyphenyl)-4, 5-dihydro-1H-1, 2, 3-triazol-4-yl] methanone(4c)

Colour: yellowish solid. M.P. $111-113{ }^{\circ} \mathrm{C}$; Yield: 74.9\%, IR: IR (KBr): $1674 \mathrm{~cm}^{-1}(\mathrm{C}=\mathrm{O}), 1630-1575$ $\mathrm{cm}^{-1}(\mathrm{~N}=\mathrm{N}), 1035 \mathrm{~cm}^{-1}(\mathrm{Ar}-\mathrm{Cl}), 1391 \mathrm{~cm}^{-1}\left(\mathrm{Ar}_{-} \mathrm{OCH}_{3}\right),{ }^{1} \mathrm{H}-\mathrm{NMR}\left(\mathrm{CDCl}_{3}\right) ; \delta(\mathrm{ppm}): 2.3(\mathrm{~s}, 1 \mathrm{H}, \mathrm{NH}) ; 3.3$ (d, 2H, $\mathrm{CH}_{2}$, adj. $\left.\mathrm{N}=\mathrm{N}\right) ; 3.71-3.72\left(\mathrm{bs}, 15 \mathrm{H}, \mathrm{OCH}_{3}\right), 4.4\left(\mathrm{~d}, 2 \mathrm{H}, \mathrm{CH}_{2}\right.$, adj. $\left.\mathrm{NH}\right)$ and $6.43-7.38(\mathrm{~m}, 5 \mathrm{H}$, Ar.-H) ${ }^{13} \mathrm{C}-\mathrm{NMR}\left(\mathrm{CDCl}_{3}\right)$; $\delta$ (ppm): 56.1, 60.8, 69.9, 81.1, 102.1, 111.0, 121.0, 131.0, 131.5, 134.7, 137.2, 137.8, 152.7, 158.5, 197.6. Mass $(\mathrm{m} / \mathrm{z})$ : 405.11, Elemental Analysis (\%): For $\mathrm{C}_{19} \mathrm{H}_{23} \mathrm{ClN}_{3} \mathrm{O}_{5}$, Calculated: C, 56.23; H, 9.97; Cl, 8.74; N, 10.35; O, 19.75. Found: C, 56.29; H, 9.99; Cl, 8.70; N, 10.31; O, 19.71 .

E (3, 4 -dihydroxyphenyl) [5-(3, 4, 5-trimethoxyphenyl)-4, 5-dihydro-1H-1, 2, 3-triazol-4-yl] methanone(4d)

Colour: White solid. M.P. $104-106{ }^{\circ} \mathrm{C}$; Yield: 72.8\%, IR: IR (KBr): $1665 \mathrm{~cm}^{-1}(\mathrm{C}=\mathrm{O}), 3540-3595 \mathrm{~cm}^{-}$ ${ }^{1}(\mathrm{Ar}-\mathrm{OH}), 1630-1575 \mathrm{~cm}^{-1}(\mathrm{~N}=\mathrm{N}),{ }^{1} \mathrm{H}-\mathrm{NMR}\left(\mathrm{CDCl}_{3}\right) ; \delta(\mathrm{ppm}): 2.3(\mathrm{~s}, 1 \mathrm{H}, \mathrm{NH}) ; 3.3\left(\mathrm{~d}, 2 \mathrm{H}, \mathrm{CH}_{2}\right.$, adj. $\mathrm{N}=\mathrm{N})$; 3.71-3.72 (bs, 9H, $\left.\mathrm{OCH}_{3}\right), 4.4\left(\mathrm{~d}, 2 \mathrm{H}, \mathrm{CH}_{2}\right.$, adj $\left.\mathrm{NH}\right) ; 6.31-7.23(\mathrm{~m}, 5 \mathrm{H}, \mathrm{Ar}-\mathrm{H}), 9.48(\mathrm{~s}, 2 \mathrm{H}, \mathrm{Ar}-$ $\mathrm{OH}),{ }^{13} \mathrm{C}-\mathrm{NMR}\left(\mathrm{CDCl}_{3}\right) ; \delta(\mathrm{ppm}): 56.1,60.8,69.9,81.1,102.1,114.4,121.3,128.4,131.9,131.1,137.2$, 137.8, 152.7, 156.5, 197.6. Mass (m/z): 373.13, Elemental Analysis (\%): For $\mathrm{C}_{18} \mathrm{H}_{19} \mathrm{~N}_{3} \mathrm{O}_{6}$, Calculated: C, 57.91; H, 5.13; N, 11.25; O, 25.71. Found: C, 57.85; H, 5.10; N, 11.31; O, 25.74. 
E (3-aminophenyl) [5-(3,4, 5-trimethoxyphenyl)-4, 5-dihydro-1H-1, 2, 3-triazol-4-yl] methanone(4e) Colour: Brown solid. M.P. $110-112{ }^{\circ} \mathrm{C}$; Yield: 74.1\% , IR: IR (KBr): $1667 \mathrm{~cm}^{-1}(\mathrm{C}=\mathrm{O}), 3415-3510 \mathrm{~cm}^{-}$ ${ }^{1}\left(\right.$ Ar. $\left.-\mathrm{NH}_{2}\right), 1630-1575 \mathrm{~cm}^{-1}(\mathrm{~N}=\mathrm{N}),{ }^{1} \mathrm{H}-\mathrm{NMR}\left(\mathrm{CDCl}_{3}\right) ; \delta(\mathrm{ppm}): 2.3(\mathrm{~s}, 1 \mathrm{H}, \mathrm{NH}) ; 3.3\left(\mathrm{~d}, 2 \mathrm{H}, \mathrm{CH}_{2}\right.$, adj. $\mathrm{N}=\mathrm{N}) ; 3.71-3.72$ (bs, 9H, $\left.\mathrm{OCH}_{3}\right), 4.4\left(\mathrm{~d}, 2 \mathrm{H}, \mathrm{CH}_{2}\right.$, adj. $\left.\mathrm{NH}\right) ; 5.28$ (s, 2H, Ar- $\left.\mathrm{NH}_{2}\right) ; 6.65-7.49$ (m, 5H, Ar.-H) ${ }^{13} \mathrm{C}-\mathrm{NMR}\left(\mathrm{CDCl}_{3}\right) ; \delta(\mathrm{ppm}): 56.1,60.8,69.9,81.1,102.1,113.1,118.8,119.4,129.1,137.5$, 137.8, 148.3, 152.7, 197.6. Mass (m/z): 356.15, Elemental Analysis (\%): For $\mathrm{C}_{18} \mathrm{H}_{20} \mathrm{~N}_{4} \mathrm{O}_{4}$, Calculated: C, 60.66; H, 5.66; N, 15.72; O, 17.96. Found: C, 60.60; H, 5.65; N, 15.72; O, 18.03 .

\section{E (3-nitrophenyl) [5-(3, 4, 5-trimethoxyphenyl)-4, 5-dihydro-1H-1, 2, 3-triazol-4-yl]methanone(4f)}

Colour: Pale yellow solid. M.P. $92-94{ }^{\circ} \mathrm{C}$; Yield: 73.6\% , IR: IR $(\mathrm{KBr}): 1667 \mathrm{~cm}^{-1}(\mathrm{C}=\mathrm{O}), 1350-1555$ $\mathrm{cm}^{-1}\left(\right.$ Ar. $\left.-\mathrm{NO}_{2}\right), 1630-1575 \mathrm{~cm}^{-1}(\mathrm{~N}=\mathrm{N}),{ }^{1} \mathrm{H}-\mathrm{NMR}\left(\mathrm{CDCl}_{3}\right) ; \delta(\mathrm{ppm}): 2.3(\mathrm{~s}, 1 \mathrm{H}, \mathrm{NH}) ; 3.3\left(\mathrm{~d}, 2 \mathrm{H}, \mathrm{CH}_{2}\right.$, adj $\mathrm{N}=\mathrm{N}) ; 3.71-3.72\left(\mathrm{bs}, 9 \mathrm{H}, \mathrm{OCH}_{3}\right), 4.4\left(\mathrm{~d}, 2 \mathrm{H}, \mathrm{CH}_{2}\right.$, adj $\left.\mathrm{NH}\right) ; 6.65-8.44(\mathrm{~m}, 5 \mathrm{H}, \mathrm{Ar}-\mathrm{H}) .{ }^{13} \mathrm{C}-\mathrm{NMR}$ $\left(\mathrm{CDCl}_{3}\right) ; \delta$ (ppm): 56.1, 60.8, 69.9, 81.1, 102.1, 123.8, 128.3, 129.6, 132.2, 135.7, 136.3, 137.2, 137.8, 147.5, 152.7, 197.6. Mass (m/z): 386.12, Elemental Analysis (\%): For $\mathrm{C}_{18} \mathrm{H}_{18} \mathrm{~N}_{4} \mathrm{O}_{6}$, Calculated: C, 55.96; H, 4.70; N, 14.50; O, 24.85. Found: C, 55.90; H, 4.68; N, 14.55; O, 24.96.

\section{Antifungal Activity}

The in vitro antifungal activity was carried out against seven fungi by Agar well diffusion method. Compounds 4(a-f) have been tested for their antifungal activity against Trichodermaharzianum, Aspergillusniger, Colletotrichumcapsici, Aspergillus tamari, Aspergillusflavus, Alternariasolani, and Penicilliumoxalicum, at $100 \mu \mathrm{g} / \mathrm{ml}$ in chloroform. Potato dextrose agar plates were seeded with the test fungi into the surface of the medium uniformly spread using sterile cotton bud. About $50 \mu \mathrm{l}(500 \mu \mathrm{g} / \mathrm{well})$ of each compound was loaded into the well $(6 \mathrm{~mm}$ diameter $)$ made by sterile cork borer, then the plates were covered with par film incubated at $27^{\circ} \mathrm{C}$ for 7 days. Nystatin and sterile distilled water were used as positive and negative control all over the experiment of $50 \mu \mathrm{g}$ concentration.

Table-1: Antimicrobial Activity of the Synthesized Compounds 4(a-f)

\begin{tabular}{c|c|c|c|c|c|c|c|c|c}
\hline S. No. & $\begin{array}{c}\text { Name of the } \\
\text { microorganism }\end{array}$ & $4 \mathrm{a}$ & $4 \mathrm{~b}$ & $4 \mathrm{c}$ & $4 \mathrm{~d}$ & $4 \mathrm{e}$ & $4 \mathrm{f}$ & Nystatin & $\begin{array}{c}\text { Distilled } \\
\text { water }\end{array}$ \\
\cline { 3 - 10 } & & & & & & & & & \\
\hline 1 & Trichodermaharzianum & 11 & 15 & 13 & 18 & 17 & 14 & 11 & 10 \\
\hline 2 & Aspergillusniger & 13 & 15 & 17 & 19 & 16 & 16 & 13 & 12 \\
\hline 3 & Collectotrichumcapsici & 09 & 14 & 15 & 17 & 15 & 13 & 12 & 09 \\
\hline 4 & Aspergillustammari & 17 & 20 & 17 & 22 & 18 & 21 & 16 & 11 \\
\hline 5 & Aspergillusflavus & 18 & 21 & 24 & 22 & 17 & 24 & 18 & 13 \\
\hline 6 & Alternriasolani & 23 & 25 & 22 & 26 & 21 & 21 & 20 & 11 \\
\hline 7 & Penicilliumoxalicum & 17 & 19 & 18 & 23 & 13 & 20 & 21 & 12 \\
\hline
\end{tabular}

\section{RESULTS AND DISCUSSION}

Chalcone 3(a-f) were prepared in good yields by Claisen Schmidt condensation reaction of substituted acetophenone 1(a-f) with 3, 4, 5-trimethoxybenzaldehyde in the presence of sodium hydroxide as a base and the water-ethanol mixture as solvent. Substituted chalcone containing $\alpha$, $\beta$-unsaturated compounds 3(a-f) were prepared in good yields. We considered this substituted chalcone as intermediate compounds for our target compounds, triazole substituted chalcone 4(a-f). These compounds were prepared by using sodium azide in methanol/AcOH using zinc metal as a catalyst and the mixture was stirred at $110^{\circ} \mathrm{C}$ (under an air atmosphere) then followed by acid hydrolysis. The formation of the product was checked by TLC. After the completion of the reaction, the mixture was poured into water and extracted with ethyl acetate, washed with $\mathrm{Na}_{2} \mathrm{CO}_{3}$ solution, dried with anhydrous $\mathrm{Na}_{2} \mathrm{SO}_{4}$, Then, the solvent evaporated to dryness, to give crude product 4(a-f). The products were recrystallized using ethanol. The structure of triazolechalcone was confirmed by IR, ${ }^{1} \mathrm{H}$ NMR and mass spectral data. The synthesized compounds were conducted for microbial activity. The results were summarized in Table-1. 


\section{CONCLUSION}

We succeeded in the synthesis of triazole derivatives of substituted chalcones and their microbial activities. Most of the compounds we synthesized were very active towards the tested microorganism compare to the standard which is already available in the market.

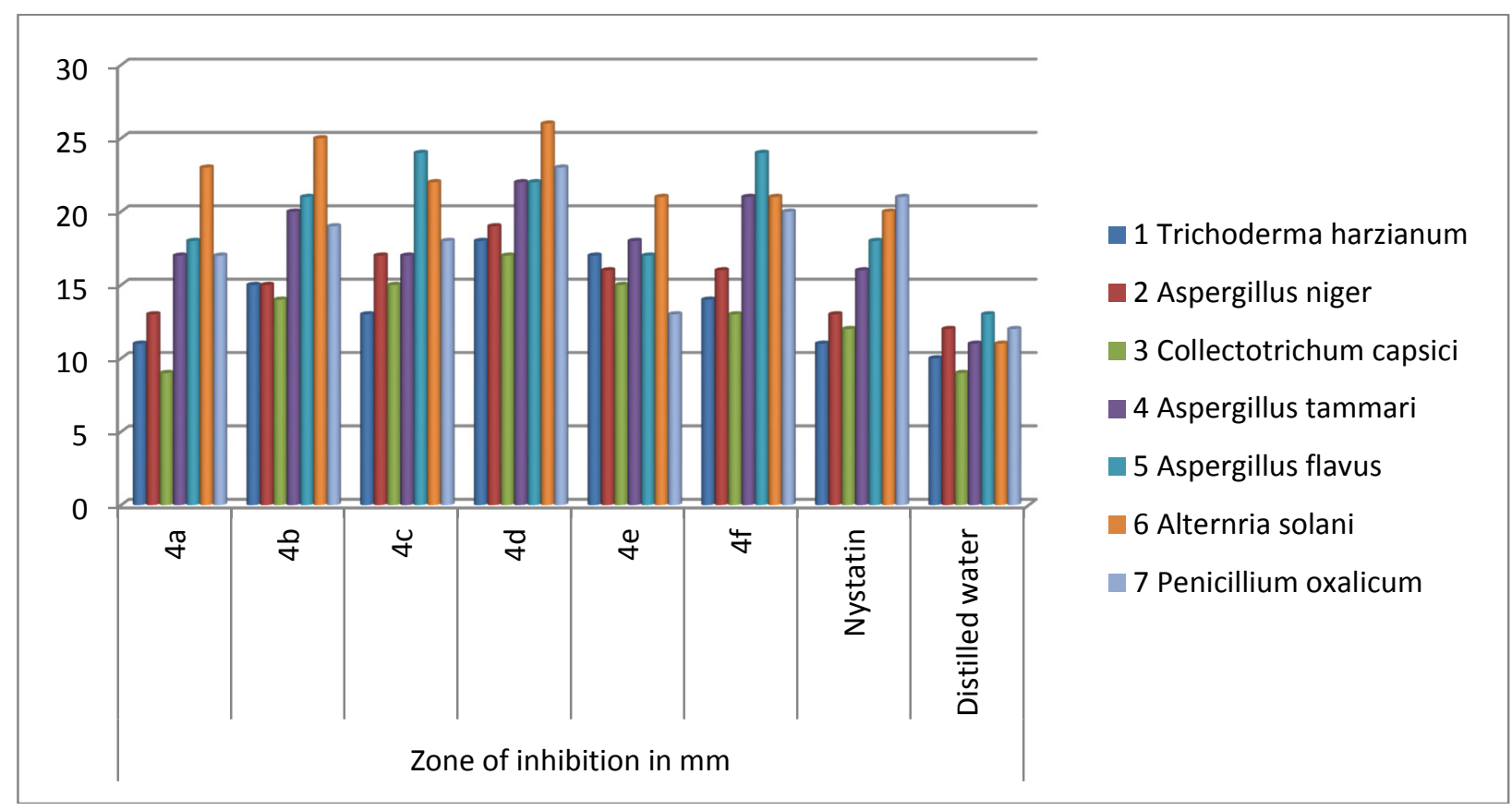

Fig-2: Graphical representation of antimicrobial activeness of synthesized compounds 4(a-f)

\section{ACKNOWLEDGMENT}

The authors are thankful to the director, CDRI, Lucknow and IIT, Chennai, for providing NMR, Mass spectra and elemental analysis data for research compounds.

\section{REFERENCES}

1. M. Chaitramallu, Devaraju, P. Ranjini, Indian Journal of Advances in Chemical Science, 2, 61(2014)

2. Begum, EVRANOSAKSOZ, Rahmiya ERTAN, FABAD J. Pharma. Sci., 36, 223(2011)

3. Alka N. Choudhary, Vijayn Juyal, International Journal of Pharmacy and Pharmaceutical Science, $\mathbf{3}$, 125(2011)

4. M. Chaitramallu, Devaraju, C. Dakshayini, P. Ranjini, Indian Journal of Advances in Chemical Science, 4(1),1(2016)

5. Lincy, Joseph, Mathew George and Jositta V Johnson, The Pharma Innovation Journal 6(2), 1(2017)

6. R. Kalirajan, S.U.Sivakumar, S. Jubie, B. Gowramma and B. Suresh, International Journal of ChemTech Research, 1(1), 27(2009)

7. Balkrishna Tiwari, AS Pratapwar, AR Tapas, SR Butle and BS Vatkar, International Journal of ChemTech Research, 2(1), 499(2010)

8. Chatana B Patil, S K Mahajan, Suvarna A Katti, Journal of Pharmaceutical Science and Research, 1(3), 11(2009)

9. Shaik Khadar Yazdan, D. Vidya Sagar and Afzal Basha Shaik, Organic \& Medicinal Chemistry International Journal, 1,1(2015)

10. Mohamed J. Elarfi and Hussniyia A. Al-Difar, Scientific Review \& Chemical Communications, 2(2), 103(2012)

11. Nidhi singh Shamim Ahmad, M. Shamsher Alam, International Journal of Pharmaceutical \& Biological Archives, 3(6), 1298(2012) 
12. Yunfeng Chen, Gang Nie, Qi Zhang, Shan Ma, Huan Li, and Qinquan Hu, Organic Letters, 17, 1118(2015), DOI: $10.1021 / \mathrm{ol} 503687 \mathrm{~W}$

13. Maya Shankar Singh, Sushobhan Chowdhury, Suvajit Koley, Tetrahedron, 72, 5257(2016), DOI: 10,1016/j.tet.2016,07.044

14. Kenneth R. Henery, Tetrahedron Letters, 7, 801(1968)

15. Nataliya Belskaya, Julia Subbotina, and Svetlana Lesogorova, Top Heterocycl Chem., 40, 51(2015), DOI: 10.1007/7081_2014_125

16. Veda Prachayasittikul and et al, Spingerplus, 4, 571(2015)

17. Graziele D. da Silva, Marina G. da Silva, Estrela M. P. V. E. Souza, Andersson Barison, Sarah C. Simões, Fernando P. Varotti, Leandro A. Barbosa, Gustavo H. R. Viana and José A. F. P. Villar, Journal of Molecules, 17, 10331(2012), DOI:10.3390/molecules170910331

[RJC-3014/2018] 